\title{
ARTICLE Neural responses to cues paired with methamphetamine in healthy volunteers
}

\author{
Kathryne Van Hedger ${ }^{1}$, Sarah K. Keedy ${ }^{1}$, Leah M. Mayo ${ }^{2}$, Markus Heilig ${ }^{2}$ and Harriet de Wit ${ }^{1}$
}

\begin{abstract}
Drug cues, or conditioned responses to stimuli paired with drugs, are widely believed to promote drug use. The acquisition of these conditioned responses has been well characterized in laboratory animals: neutral stimuli paired with drugs elicit conditioned responses resembling the motivational and incentive properties of the drug itself. However, few studies have examined acquisition of conditioning, or the nature of the conditioned response, in humans. In this study, we used fMRI to examine neural responses to stimuli that had been paired with methamphetamine or placebo in healthy young adults. Participants first underwent four conditioning sessions in which visual-auditory stimuli were paired with either methamphetamine ( $20 \mathrm{mg}$, oral) or placebo. Then on a drug-free test day, the stimuli were presented during an fMRI scan to assess neural responses to the stimuli. We hypothesized that the stimuli would elicit drug-like brain activity, especially in regions related to reward. Instead, we found that the methamphetamine-paired stimuli, compared to placebo-paired stimuli, produced greater activation in regions related to visual and auditory processing, consistent with the drug's unconditioned effects on sensory processing. This is the first study to demonstrate conditioned neural responses to drug-paired stimuli after just two pairings of methamphetamine in healthy adults. The study also illustrates that conditioned responses may develop to unexpected components of the drug's effects.
\end{abstract}

Neuropsychopharmacology (2018) 43:1732-1737; https://doi.org/10.1038/s41386-017-0005-5

\section{INTRODUCTION}

Conditioned responses to stimuli paired with drugs, or drug cues, are known to promote drug use in animal models and human drug users [1-4]. In animal models, drug cues increase operant responding previously reinforced with cocaine, nicotine, amphetamine, or heroin $[2,5-7]$, and in humans, drug cues increase craving for cigarettes, alcohol, and other drugs [8,9]. Indeed, drug cues retain their ability to elicit drug-seeking behavior long after cessation of drug use [10-12]. Decades of research indicates that, in laboratory animals, drug cues acquire motivational properties through Pavlovian conditioning [4]. In contrast, we know less about the acquisition of conditioned drug effects in humans. Studies investigating drug cues in humans typically utilize generic drug-related stim prior contiguity with the drug stimulus is mostly inferred, without explicit knowledge of how the responses were acquired $[13,14]$. Recently, we developed a procedure to study the acquisition of conditioned drug responses in healthy young adults, showing that an initially neutral stimulus (e.g., an audiovisual cue) paired with a drug (e.g., amphetamine or alcohol) acquires motivational and incentive properties of the drug itself [15-17].

With the ability to control the process of acquisition of conditioned drug responses in humans, we can now investigate the nature of the conditioned responses and the neurobiological mechanisms underlying responses to cues. Conditioned responses to cues have been reported using different measures, such as subjective reports of 'liking' or 'preferring' the conditioned stimulus [18], behavioral indices of emotion (e.g., facial electromyography $[15,16])$, attention to the stimulus (e.g., eye movements $[15,16,19])$, striatal dopamine release using PET [20] and neural activity using functional magnetic resonance imaging (fMRI) measures [21]. The sensitivity of these indices varies across studies [22], and the outcome measures do not always co-vary [16]. Why certain components of a drug's effects become conditioned while others do not is unknown. The nature of the conditioned response may reflect procedural variations across studies, differences in sensitivity of measurement, or differences in brain or behavioral processes underlying conditioning.

Brain imaging studies in humans provide a novel way to study drug conditioning and the nature of conditioned responses. In this study we used fMRI to examine neural responses to a stimulus that had been paired de novo with a stimulant drug. Because conditioned responses typically resemble the unconditioned drug response $[4,23,24]$, we would expect the conditioned neural response to a stimulant-paired cue to mirror the direct drug effect. Stimulant drugs increase activity in the mesolimbic dopamine system, commonly referred to as a reward circuit (e.g., refs. [25, 26]). Interestingly, stimulant drugs also affect brain activity in areas related to sensory processing, raising the possibility that sensory effects could also become conditioned [27, 28]. In this study we examined brain activation while viewing compound stimuli (visual images of a mountain or ocean scene accompanied by appropriate sounds of birds or waves) that had been previously paired two times each with oral methamphetamine or placebo. The scans occurred during a separate session several days after the last conditioning session, while subjects were drug-free. We examined BOLD responses to the stimuli using fMRI. We hypothesized that subjects would exhibit larger BOLD responses to the drug-paired

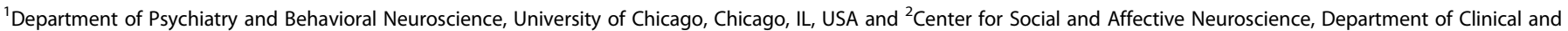
Experimental Medicine, Linköping University, Linköping, Sweden

Correspondence: Harriet de Wit (hdew@uchicago.edu)

Received: 6 November 2017 Accepted: 20 December 2017

Published online: 30 January 2018 
cue, compared to the placebo-paired cue, in brain regions related to reward circuitry (e.g., ventral striatum) and sensory function.

\section{METHODS AND MATERIALS}

Participants

Healthy men and women $(N=59)$ aged $18-35$ years were recruited via flyers and online advertisements. Respondents were excluded if they had a current psychiatric disorder (DSM 5; APA 2013), past year substance dependence, current use of prescription medication (excluding hormonal birth control), abnormal electrocardiogram, night shift work, left-handedness, less than high school education, lack of fluency in English, consuming more than four alcohol-containing or caffeine-containing drinks per day, body mass index outside the range $19-26 \mathrm{~kg} / \mathrm{m}^{2}$, and contraindications for fMRI scanning (e.g., claustrophobia, copper IUD). Of the 59 participants who enrolled, four did not complete the fMRI scan, two were excluded for falling asleep during the fMRI scan, and two were excluded for excessive movement during the fMRI scan, leaving a final study sample of 51 participants. This study was approved by the University of Chicago Biological Sciences Division Institutional Review Board.

\section{Study Design}

This study used a within-subjects design with four conditioning sessions followed by an imaging session. During the conditioning sessions participants received either methamphetamine $(20 \mathrm{mg}$ oral) or placebo, each paired with distinctive audiovisual cues consisting of a mountain scene with bird sounds or an ocean scene with wave sounds. Cues and drug order were counterbalanced. Preferences for the two cues were assessed before and after the conditioning, using measures of behavioral preference, subjective liking, and attentional bias. During the final fMRI session (no drug administration), we examined patterns of brain activation to the methamphetamine-paired and placebo-paired cues.

\section{Session Procedures}

The procedures used for this study are described in detail in ref. [15]. Our modification included adding an fMRI scan to the post conditioning session, and we did not collect data on emotional reactivity to the cues. Otherwise the procedures were identical.

\section{Drug}

Methamphetamine (Desoxyn, Lundbeck) was crushed and mixed with $10 \mathrm{ml}$ of combined Ora-Plus and Ora-Sweet syrups (Paddock Laboratories, Minneapolis, MN [15]). We have previously reported conditioned effects with this dose [15, 22]. Placebo consisted of $10 \mathrm{ml}$ of equal parts Ora-Plus and Ora-Sweet. Syrups were administered in clear 1 oz plastic cups.

Drug Effects Measures

Subjective Measures. Subjective effects were assessed using the Drug Effects Questionnaire (DEQ [29, 30]), and the Profile of Mood States (POMS [31]). Measurements were taken before and 15, 30, 70,115 , and $200 \mathrm{~min}$ after drug administration.

Cardiovascular Measures. Heart rate (HR) and blood pressure (BP) were monitored (Omron, Lake Forest, IL) during the conditioning sessions at the same intervals as the subjective measures.

\section{Conditioning Measures}

Participants completed the same behavioral preference, subjective liking and attentional bias tasks described by [15] with minor modifications detailed below.

Behavioral Preference Task. For the behavioral preference task participants were given a choice between images containing the methamphetamine- and placebo-paired cues and selected the one they preferred. During the post-conditioning session participants completed this task twice (i.e., 30 trials) while in the scanner. However for consistency, behavioral data from only the first 15 trials were used to compare to pre-test.

Attentional Bias Task. Attentional bias was measured using a modified dot-probe task with images of the two cues [15, 32]. In addition to the previous outcome measures of orienting and sustained attention we also measured reaction time (RT) to the probe.

fMRI Task. fMRI data were obtained while subjects completed a modified version o forced choice preference task, and neural activation was recorded while subjects experienced the methamphetamine-paired and placebo-paired cues. The task consisted of 30 trials. Each trial was comprised of $11.2 \mathrm{~s}$ of each cue plus task image from the conditioning sessions, with the matched soundtrack. Subjects were instructed to passively view and listen. Trials alternated between ocean and mountain backgrounds. Then both images were shown side by side, and participants indicated which image they preferred using a button box in their right hand. The task varied slightly in length because it was self-paced, but it lasted about $18 \mathrm{~min}$. The cues were presented for the same amount of time for all participants.

Imaging was done on a Philips Achieva 3.0 T scanner using a 32channel headcoil and a gradient-echo echo-planar imaging sequence with acquisition parameters $\mathrm{TR}=2000$; $\mathrm{TE}=30 ; 36$ $3.75 \mathrm{~mm}$ thick axial slices aligned to the AC-PC line, no gap; $24 \times 24 \mathrm{~cm}$ FOV, $\left(3.75 \mathrm{~mm}^{3}\right.$ voxels); SENSE factor $=2$, Flip angle $=$ $90^{\circ}$. Four images were acquired and discarded just prior to task start. A high resolution T1-weighted image (MPRAGE sequence) was also acquired to assess for incidental findings, and for alignment and spatial standardization of the functional data. Subject head motion was minimized with foam packing around the head. Stimuli were viewed via projection onto a mirror system mounted on the headcoil. Sounds were delivered via earbud headphones and played at $\sim 80 \mathrm{~dB}$.

\section{Subject-Level Functional Image Processing}

Neuroimaging data analysis was conducted with AFNI [33]. Preprocessing steps included alignment of the time series to the last image (as it was closest in time to acquisition of the structural image), spatial registration of the aligned time series data to the anatomical scan, anatomical scan warping to Talairach space and warp applied to functional data, functional data spatially smoothed with a $4 \mathrm{~mm}$ fwhm Gaussian kernel, and intensity normalization. With this preprocessed data, neural activation was then estimated for each subject to the methamphetamine- and placebo-paired cues using a linear regression analysis as implemented in AFNI's 3dDeconvolve program. De-meaned and derivatives of motion parameters were estimated and included as covariates of no interest. Viewing/listening to the methamphetamine-paired and placebo-paired cues were modeled as block functions. No other aspect of the imaging task (e.g., the choice-making) was of interest for analysis. Mean activation forired cues were extracted from bilateral ventral striatum for each subject for primary analysis of impact of drug-associated conditioning of reward systems. The mask for ventral striatum was that of the "7 subdivisions" striatum probabilistic atlas of ref. [34].

\section{Statistical Analysis}

Drug Effects. We used repeated-measures analysis of variance (RM-ANOVA) to examine subjective and cardiovascular effects during the two methamphetamine sessions and the two PBO sessions. Time (before and 15, 30, 70, 115, and $200 \mathrm{~min}$ after drug administration) and drug (methamphetamine and placebo) were treated as within-subjects factors. To explore relationships 
between subjective drug effects (e.g., drug liking) and our conditioning measures, we calculated peak change scores from baseline averaging across the two methamphetamine sessions and across the two placebo sessions to create single scores for methamphetamine and placebo.

Behavioral Effects of Conditioning. Our behavioral index of conditioning was the difference in responses to the audiovisual cues at pre-test and post-test on the forced choice task, subjective liking task, and attentional bias task. For each task we conducted RM-ANOVA with time and drug as within-subjects factors.

Imaging. Analyses were conducted as within subject comparisons, directly contrasting the methamphetamine-paired and placebo-paired cues, using age, sex, and methamphetaminepaired cue (whether ocean or mountain was the methamphetamine-paired cue) as covariates. Parameter estimates for extracted ventral striatum activation were compared between methamphetamine-paired and placebo-paired cues using paired $t$-tests. Secondary whole-brain analyses were conducted using AFNl's 3dmvm, implementing an ANCOVA with sex, age, methamphetamine-paired cue as covariates and comparing methamphetamine- versus placebo-paired cue activation on a voxelwise basis. Significant clusters of voxels were identified if they met a $p>.001$ familywise error correction threshold, where at least 20 contiguous voxels each met $p>.001$. This threshold was determined using 3dClustSim, which ran 10,000 Monte Carlo simulations of randomly generated data of the same resolution and in-brain inclusion mask (34,990 3.5 $\mathrm{mm}^{3}$ voxels) as the preprocessed data, and utilizing the average spatial autocorrelation function of the subjects (obtained via 3dFWHMx). These procedures avoid concerns around high falsepositive rates in fMRI [35]. Finally, Pearson correlations were conducted between behavioral data and activation in bilateral ventral striatum and any other regions identified by the wholebrain analysis showing differences for methamphetamine- versus placebo-paired cues.

\section{RESULTS}

Participants

Table 1 summarizes the demographic characteristics and drug use patterns of the 51 participants who completed the study.

Drug Effects During Conditioning Sessions

Subjective Measures. Subjective responses to methamphetamine did not differ across the first and second drug sessions, thus responses for the two methamphetamine sessions and two placebo sessions were averaged. As expected, methamphetamine increased ratings of feel drug, like drug, feel high, and want more compared to placebo (Table 2). Methamphetamine also increased feelings of friendliness, anxiety, elation, and vigor, and decreased feelings of fatigue and confusion (POMS). These results are in line with previous studies from this laboratory [15, $17,36,37]$.

Cardiovascular Measures. The cardiovascular effects of methamphetamine versus placebo did not differ across the two administrations, so the two methamphetamine and two placebo sessions were averaged. Methamphetamine significantly increased both blood pressure (reported here as mean arterial pressure) and heart rate compared to placebo (Table 2). Again, these results are similar to those previously reported [15].

Behavioral Effects on Post-Conditioning Test Session Forced Choice Task. Preference for the methamphetamine-paired versus placebo-paired cues did not change from before to after
Table 1. Demographic information and nonmedical drug use $(N=51)$. Mean values for current drug use are reported from only current users, and number of current users is indicated in parentheses

\begin{tabular}{ll}
\hline & Percent (N) or Mean (SEM) \\
\hline Gender & \\
Male/Female & $25 / 26$ \\
Age (years) & $23.5(0.6)$ \\
Education (years) & $14.7(0.3)$ \\
BMI & $22.9(0.2)$ \\
Race & \\
Caucasian & $51.0 \%(26)$ \\
African-American & $21.6 \%(11)$ \\
Asian & $7.8 \%(4)$ \\
Other & $15.7 \%(8)$ \\
Unknown & $3.8 \%(2)$ \\
Current drug use & \\
Caffeinated drinks per day $(n=33)$ & $1.3(0.1)$ \\
Cigarettes per week $(n=9)$ & $10.3(2.9)$ \\
Alcoholic drinks per week $(n=46)$ & $7.7(0.6)$ \\
Lifetime drug use (ever used) & \\
Marijuana & $82.4 \%(42)$ \\
Opiates & $25.5 \%(13)$ \\
Stimulants & $45.1 \%(23)$ \\
Hallucinogens & $49.0 \%(25)$ \\
MDMA & $33.3 \%(17)$ \\
Sedatives & $29.4 \%(15)$ \\
&
\end{tabular}

Table 2. Subjective and cardiovascular effects of methamphetamine (MA) and placebo (PBO; $N=51$ )

\begin{tabular}{|c|c|c|c|c|c|}
\hline \multirow[b]{2}{*}{ Subjective Effects } & \multicolumn{2}{|l|}{ PBO } & \multicolumn{2}{|l|}{ MA } & \multirow[b]{2}{*}{$T$-value } \\
\hline & Mean & SEM & Mean & SEM & \\
\hline \multicolumn{6}{|l|}{$D E Q$} \\
\hline Feel & 19.86 & 3.09 & 50.39 & 3.30 & $9.81^{* * *}$ \\
\hline Like & 23.77 & 2.94 & 68.85 & 3.33 & $12.55^{* * *}$ \\
\hline Dislike & 32.47 & 4.23 & 34.90 & 3.31 & 0.61 \\
\hline High & 11.21 & 2.22 & 42.05 & 3.79 & $9.50^{* * *}$ \\
\hline More & 20.38 & 2.90 & 65.12 & 3.99 & $11.31^{* * * *}$ \\
\hline \multicolumn{6}{|l|}{ POMS } \\
\hline Friendliness & -2.06 & 0.50 & 2.21 & 0.70 & $5.63^{* * *}$ \\
\hline Anxiety & -0.27 & 0.33 & 1.68 & 0.66 & $2.71^{* *}$ \\
\hline Elation & -1.24 & 0.30 & 2.78 & 0.59 & $6.43^{* * *}$ \\
\hline Anger & 0.33 & 0.36 & 0.28 & 0.51 & -0.10 \\
\hline Fatigue & 1.04 & 0.49 & -1.74 & 0.59 & $-3.97^{* * *}$ \\
\hline Depression & -0.36 & 0.48 & -0.03 & 0.39 & 0.50 \\
\hline Confusion & 0.36 & 0.28 & -0.52 & 0.34 & $-2.16^{*}$ \\
\hline Vigor & -2.10 & 0.58 & 4.89 & 0.84 & $7.31^{* * *}$ \\
\hline \multicolumn{6}{|c|}{ Cardiovascular effects } \\
\hline Blood pressure & -4.04 & 1.21 & 10.95 & 1.38 & $8.74^{* * *}$ \\
\hline Heart rate & -5.32 & 1.58 & 7.43 & 2.43 & $6.07^{* * * *}$ \\
\hline $\begin{array}{l}\text { DEQ drug effect } \\
\text { and POMS value } \\
\text { two MA session } \\
{ }^{*} p<.05,{ }^{* *} p<.0\end{array}$ & onr & $M$ & of & sta & $\begin{array}{l}\text { te: DEQ } \\
\text { PBO and } \\
\text { pressure }\end{array}$ \\
\hline
\end{tabular}




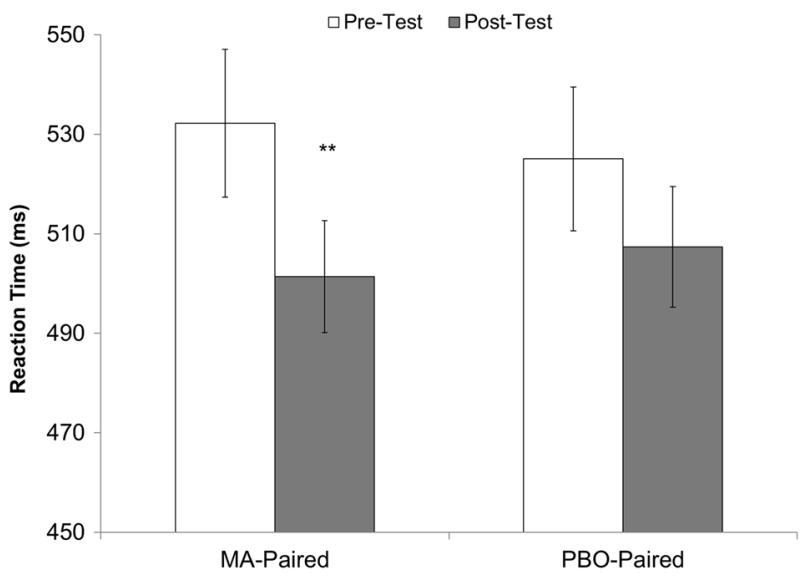

Fig. 1 Reaction times on a modified dot-probe task with the methamphetamine-paired and placebo-paired cues (attentional bias) before and after conditioning. Participants responded significantly faster to the probe when it appeared in the same location as the methamphetamine-paired cue following conditioning. ${ }^{* *} p=.01$

conditioning (mean preference before $4.37 \pm 0.33$ SEM and after $4.47 \pm 0.33$ SEM).

Subjective Liking Task. Liking ratings of both methamphetaminepaired and placebo-paired cues declined from before to after conditioning (time, $F(1,50)=30.8, p<.001$ ). Before conditioning mean liking of to-be-methamphetamine-paired cues was 78.97 \pm 2.47 SEM, and to-be-placebo-paired cues was $82.89 \pm 2.04$ and after conditioning liking of methamphetamine cues was $71.67 \pm$ $2.93 \mathrm{SEM}$, and placebo cues was $70.71 \pm 3.03$ ). There was no main effect for drug or interaction with drug.

Attentional Bias Task: After conditioning, RT's were significantly faster, $F(1,50)=4.61, p<.05$ for both stimuli. However, participants were faster to respond to the probe when it appeared in the same location as the methamphetamine-paired cue, compared to the PBO-paired cue (time by drug interaction, $F(1,50)=4.16, p<.01$; mean pre RT $501.40 \pm 11.25$ SEM and post RT 532.21 \pm 14.87 SEM; Fig. 1). The RT's for the to-be-conditioned stimuli did not differ at pre-test and RT's did not change from pre- to post-conditioning for the placebo image. Data for orienting attention and sustained attention measures were lost for 6 participants either because of equipment malfunction (5) or participant constraints (1). All remaining subjects had at least $50 \%$ of trials with valid gazes. On the measure of orienting attention there was a marginally significant interaction, $F(1,44)=3.77, p=.06$. Subjects directed slightly more initial gazes at the methamphetamine-paired cue at post-test $(18.27 \pm 0.49 \mathrm{SEM})$ compared to pre-test (16.62 \pm 0.57 SEM; Fig. 2). On the measure of sustained attention (dwell time) participants spent marginally more time looking at both cues at post-test compared to pre-test (time, $F(1,44)=3.77, p=.06$ ), but methamphetamine-paired and placebo-paired cues did not differ on this measure. There was no main effect for drug and no significant interaction

Neuroimaging results

All subjects met motion threshold correction criteria of $<3 \mathrm{~mm}$ displacement. The extracted activation during the methamphetamine- versus placebo-paired cues did not differ in the ventral striatum, but for the whole-brain analysis, activation for methamphetamine-paired cues relative to placebo-paired cues increased significantly in bilateral visual and auditory cortex, and insula (Table 3, Fig. 2). No clusters showed significantly higher activation for placebo-paired relative to methamphetamine-paired cues. Pearson correlations were conducted between behavioral

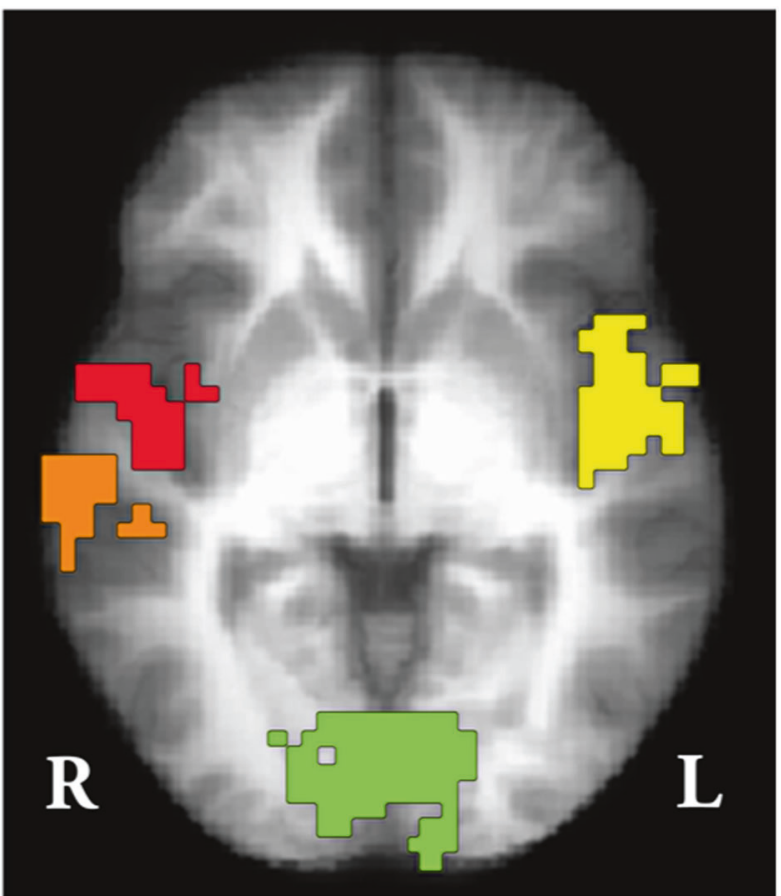

Fig. 2 Images in neurological convention (left is right). Methamphetamine-paired images evoked significantly greater activation than placebo-paired images in clusters in bilateral primary/secondary visual cortex (green), left auditory cortex and insula (yellow), and right auditory cortex (orange, red) and insula (red)

findings and extracted activation for the differences between methamphetamine- versus placebo-paired cues in ventral striatum, primary/secondary visual cortex, auditory cortex, and insula. No significant associations were found ( $F$-values $<0.2$ and $>-0.2, p$ values $>.05$ corrected).

\section{DISCUSSION}

In the present study, we used fMRI to detect conditioned responses to sensory cues paired with methamphetamine or placebo in healthy young adults [15]. After just two drug pairings, we observed greater brain activation to the methamphetaminepaired, compared to the placebo-paired stimuli, consistent with a conditioning effect. The scanning session was conducted several days after the last conditioning session, while subjects were in a drug-free state. Surprisingly, the conditioned brain activation corresponded to regions involved in visual and auditory processing, rather than reward regions.

To our knowledge, this is the first study investigating conditioning of neural responses to initially neutral drug-paired cues in healthy humans. On the basis of prior knowledge that stimulant drugs activate dopaminergic reward circuits [25], we hypothesized that the methamphetamine-paired cue would elicit activity in reward-related regions. However, the methamphetamine-paired cue did not activate regions related to reward. Instead, it activated regions involved in sensory processing. Interestingly, this is consistent with other reports from imaging studies of amphetamine responses [38] and drug cues $[27,38]$ used fMRI to show that amphetamine enhances brain responses to sensory stimuli. Consistent with these reports, we observed robust activation in visual and auditory regions (i.e., bilateral primary/secondary visual cortex, auditory cortex, and insula) upon presentation of the methamphetamine-paired stimulus, relative to the placebo-paired stimulus. The behavioral indicators of conditioning were less clear: the stimulus-drug 
Table 3. Brain areas in which drug-paired stimuli elicited greater activation than placebo-paired stimuli

Cluster size (number of $\quad$ Center of Mass $x \quad$ Center of Mass $y \quad$ Center of Mass $z \quad$ Peak $T$-value $3.5 \mathrm{~mm}^{3}$ voxels)

Bilateral cuneus and lingual gyri (BA 17, 18)
Left superior temporal gyrus and Insula
Right superior temporal gyrus
Right superior temporal gyrus and Insula

533
112
109
90

1.0
-46
61
-50

-80
-4
-31
-3

$\begin{array}{cc}2 & 8.7 \\ -1 & 6.3 \\ 6 & 5.9 \\ -3 & 6.6\end{array}$

pairings did not change behavioral preference for the cues, although there was a small increase in attentional bias toward the methamphetamine-paired stimulus. We conclude from these findings that the conditioned neural responses to the cues were related more to the arousing and attentional effects of methamphetamine than to its rewarding effects.

The Pavlovian conditioning procedure allows drug-like responses to become conditioned to neutral stimuli, but it does not provide the experimenter with control over which drug effects become conditioned [39]. Stimulant drugs, like other drugs, produce a range of effects in the brain and on behavior, any of which might become conditioned. Indeed, using this procedure we have previously reported conditioning with various measures, including liking of the drug-paired cue [22], positive emotional responses to the cue (measured by facial electromyography), behavioral preference for the cue or attentional bias toward the cue $[15,17,22]$. However, the responses that exhibited conditioning has varied across studies. In the present study one hypothesis was that the conditioned stimulus would activate the same dopamine-rich brain areas activated by methamphetamine [40], and that it would produce conditioned, reward-like responses. Instead, we observed conditioned neural activation that may be more closely related to the effects of methamphetamine on arousal, vigilance and reactivity to environmental stimuli [41-43]. Interestingly, in another example of unanticipated conditioned drug responses in humans [44], studied conditioned responses to a taste that had been repeatedly paired with the antidepressant amitriptyline or placebo. The authors detected no conditioned antidepressant-like mood effects (as measured by standardized mood rating scales), but instead found that the amitriptyline-paired taste elicited more antidepressant-like side effects such as dry mouth, dizziness and fatigue. Thus, although the functional aspects of Pavlovian conditioning can be established in a controlled study, the responses that become conditioned are not under experimental control. This has implications for understanding the role of conditioned cues in motivated, or addictive behaviors: It is not always clear which responses will be conditioned, or which come to influence future motivated behavior.

Compared to the placebo-paired cue, the methamphetaminepaired cue enhanced activity in both the visual and auditory cortices. These regions correspond directly to the visual and auditory aspects of the stimuli used, and are typically involved in passive sensory processing. As expected with a simulant drug, methamphetamine increased ratings of vigor, and increased heart rate and blood pressure during the conditioning sessions. Thus, our finding of increased activity upon presentation of the drug-paired cue reflects the combined conditioning effect of the sensory stimulation of the cue and the stimulating effect of the drug [38].

The drug-paired cue also increased the activation in the insula, suggesting that additional neural coding of salience may have contributed to this effect [45]. The posterior insula is known to receive sensory input from auditory and visual cortex [46], and may integrate this input with other sensory and motivational inputs, thus influencing the afferent reciprocal connections of insula back to motivational/limbic structures, which could reflect an early stage of stimulus salience learning. Alternatively, it is also possible that the apparent involvement of the insula is an artifact of its proximity to auditory cortex, where the signal was strong and the insula appeared to be involved because of spatial blurring.

The other conditioned effect detected in the present study was an increase in attentional bias toward the drug-paired stimuli. Attentional bias toward drug-related cues is a strong indicator of drug use in established drug users [19], and the present findings suggest that this type of attentional bias may develop early in the process of conditioning. Participants in this study exhibited faster RTs to the methamphetamine-paired cue after conditioning, and marginal increases in orienting attention to the methamphetamine-paired cue. However, we did not replicate previous findings of increases in sustained attention to the methamphetamine-paired cue and decreases in sustained attention to the placebo-paired cue [15]. This difference may be a result of the fact that post-conditioning measures were collected after the fMRI scan where the cues were presented without drug pairings. One previous study using these cues found that conditioned responses extinguish quickly when the cues are presented without drug [47]. Exploratory post hoc analysis indicated that attentional bias toward the methamphetaminepaired cue was not correlated with the magnitude of brain activation, suggesting that the conditioned increase in vigilance to the drug-paired cue was independent of the cue-induced brain activation.

This study had several limitations, including a modest sample size. It is possible that with a larger sample we might have detected conditioning on other behavioral measures, and conditioned activation in reward-related regions of the brain, consistent with the drug's euphorigenic effects. In addition, compared to a natural setting where drug users experience many pairings, our participants experienced very few pairings between the cues and the drug itself. Thus, some conditioned effects may only develop after more pairings. Further, we used a low, oral dose of methamphetamine in this study. Drug users typically use much higher doses, and administer the drugs by routes that involve faster onset. These conditions also are likely to enhance conditioning. Finally, we collected fMRI data only after conditioning, thus we do not have fMRI data on the direct effects of methamphetamine on responses to the cues. Future studies should assess neural responses to the cues at all three phases: before drug exposure, in the presence of drug, and after conditioning.

We draw several conclusions from this study. First, it is possible to establish a Pavlovian conditioned neural response to cues paired with low, oral doses of methamphetamine in healthy adults. This finding underscores the notion that classical conditioning develops extremely rapidly, supporting its key role in the development of addiction. Second, the conditioned neural responses may extend beyond the expected, dopamine-rich reward areas, to brain areas involved in sensory processing of a drug-paired cue [27]. Indeed, Jasinska et al. [27]. suggested that drugs of abuse may facilitate sensory processing of cues, thus partially explaining the increases in sensory and perceptual cortices often reported in cue imaging studies. Interestingly we found in a previous fMRI study [38] that administration of amphetamine enhanced activity in the visual cortex elicited by a visual stimulus, and enhanced activity in the motor cortex that 
was elicited by a motor task. The present study appears to have conditioned this drug-enhanced activation. It remains to be determined what role conditioned responses play in future drugseeking. By studying this early stage of acquisition of conditioning, however, we may be poised to intervene earlier with prevention or treatment strategies.

\section{ACKNOWLEDGEMENTS}

This research was supported by DA037011, and benefitted from S100D018448 awarded to the University of Chicago MRI Research Center. Kathryne Van Hedger is currently supported by NIMH training grant T32MH020065. Dr. de Wit has received support unrelated to this study from the following sources: Consulting fees from Marinius and Jazz Pharmaceuticals; gift of a study drug from Indivior; and support for a research study from Insys Therapeutics.

\section{ADDITIONAL INFORMATION}

Conflict of interest The authors declare that they have no conflict of interest.

\section{REFERENCES}

1. Berridge $\mathrm{KC}$, Robinson TE. Liking, wanting, and the incentive-sensitization theory of addiction. Am Psychol. 2016;71:670-9.

2. Perry CJ, Zbukvic I, Kim JH, Lawrence AJ. Role of cues and contexts on drugseeking behaviour. Br J Pharmacol. 2014;171:4636-72.

3. Robinson TE, Berridge KC. The neural basis of drug craving: an incentivesensitization theory of addiction. Brain Res Rev. 1993;18:247-91.

4. Stewart J, de Wit H, Eikelboom R. Role of unconditioned and conditioned drug effects in the self-administration of opiates and stimulants. Psychol Rev. 1984;91:251-68.

5. Arroyo M, Markou A, Robbins TW, Everitt BJ. Acquisition, maintenance and reinstatement of intravenous cocaine self-administration under a second-order schedule of reinforcement in rats: effects of conditioned cues and continuous access to cocaine. Psychopharmacology. 1998;140:331-44.

6. Crombag HS, Bossert JM, Koya E, Shaham Y. Context-induced relapse to drug seeking: a review. Philos Trans R Soc B Biol Sci. 2008;363:3233-43.

7. Palmatier MI, Liu X, Matteson GL, Donny EC, Caggiula AR, Sved AF. Conditioned reinforcement in rats established with self-administered nicotine and enhanced by noncontingent nicotine. Psychopharmacology. 2007;195:235-43.

8. Ehrman RN, Robbins SJ, Childress AR, O'Brien CP. Conditioned responses to cocaine-related stimuli in cocaine abuse patients. Psychopharmacology. 1992;107:523-9.

9. Monti PM, Binkoff JA, Abrams DB, Zwick WR, Nirenberg TD, Liepman MR. Reactivity of alcoholics and nonalcoholics to drinking cues. J Abnorm. 1987;96:122-6.

10. Bedi G, Preston KL, Epstein DH, Heishman SJ, Marrone GFm Shaham Y, et al. Incubation of cue-induced cigarette craving during abstinence in human smokers. Biol Psychiatry. 2011;69:708-11.

11. Meil WM, See RE. Conditioned cued recovery of responding following prolonged withdrawal from self-administered cocaine in rats: an animal model of relapse. Behav Pharmacol. 1996;7:754-63.

12. O'Brien CP, Childress AR, McLellan AT, Ehrman R. Classical conditioning in drugdependent humans. Ann N Y Acad Sci. 1992;654:400-15.

13. Robbins SJ, Ehrman RN, Childress AR, O'Brien CP. Comparing levels of cocaine cue reactivity in male and female outpatients. Drug Alcohol Depend. 1999;53:223-30.

14. Waters AJ, Carter BL, Robinson JD, Wetter DW, Lam CY, Kerst W, et al. Attentional bias is associated with incentive-related physiological and subjective measures. Exp Clin Psychopharm. 2009;17:247-57.

15. Mayo LM, de Wit H. Acquisition of responses to a methamphetamine-associated cue in healthy humans: self-report, behavioral, and psychophysiological measures. Neuropsychopharmacology. 2015;40:1734-41.

16. Mayo LM, de Wit H. Acquisition of conditioned responses to a novel alcoholpaired cue in social drinkers. J Stud Alcohol Drugs. 2016;77:317-26.

17. Mayo LM, Fraser D, Childs E, Momenan R, Hommer DW, de Wit H, et al. Conditioned preference to a methamphetamine-associated contextual cue in humans. Neuropsychopharmacology. 2013;38:921-9.

18. Childs $\mathrm{E}$, de Wit $\mathrm{H}$. Alcohol-induced place conditioning in moderate social drinkers. Addiction. 2016;111:2157-65.

19. Field M, Cox W. Attentional bias in addictive behaviors: a review of its development, causes, and consequences. Drug Alcohol Depend. 2008;97:1-20.

20. Boileau I, Dagher A, Leyton M, Welfeld K, Booji L, Diksic M, et al. Conditioned dopamine release in humans: a positron emission tomography [11C]raclopride study with amphetamine. J Neurosci. 2007;27:3998-4003.
21. Kareken DA, Grahame N, Dzemidzic M, Walker MJ, Lehigh CA, O'Connor SJ. fMRI of the brain's response to stimuli experimentally paired with alcohol intoxication. Psychopharmacology. 2012;220:787-97.

22. Cavallo JS, Mayo LM, de Wit H. Acquisition of conditioning between methamphetamine and cues in healthy humans. PLoS ONE. 2016;11:e0161541.

23. Martin-Soelch C, Linthicum J, Ernst M. Appetitive conditioning: neural bases and implications for psychopathology. Neurosci Biobehav Rev. 2007;31:426-40.

24. Pavlov, IP. Conditioned reflexes: an investigation of the physiological activity of the cerebral cortex. New York, NY: Dover Publications; 1927.

25. Breiter HC, Gollub RL, Weisskoff RM, Kennedy DN, Makris N, Berke JD, et al. Acute effects of cocaine on human brain activity and emotion. Neuron. 1997;19:591-611.

26. Volkow ND, Wang G, Fowler JS, Logan J, Gerasimov M, Maynard L, et al. Therapeutic doses of oral methylphenidate significantly increase extracellular dopamine in the human brain. J Neurosci. 2001;21:RC121.

27. Jasinska AJ, Stein EA, Kaiser J, Naumer MJ, Yalachkov Y. Factors modulating neural reactivity to drug cues in addiction: a survey of human neuroimaging studies. Neurosci Biobehav Rev. 2014;38:1-16.

28. Yalachkov Y, Kaiser J, Naumer MJ. Functional neuroimaging studies in addiction: multisensory drug stimuli and neural cue reactivity. Neurosci Biobehav Rev. 2012;36:825-35.

29. Johanson $C E$, Uhlenhuth $E H$. Drug preference and mood in humans: diazepam. Psychopharmacology. 1980;71:269-73.

30. Morean ME, de Wit H, King AC, Sofuoglu M, Rueger SY, O'Malley SS. The drug effects questionnaire: psychometric support across three drug types. Psychopharmacology. 2013;227:177-92.

31. McNair, D, Lorr, M. \& Droppleman, L. Profile of mood states. San Diego, CA: Educational and Industrial Testing Service; 1971. .

32. Wardle MC, Garner MJ, Munafó MR, de Wit H. Amphetamine as a social drug: effects of d-amphetamine on social processing and behavior. Psychopharmacology. 2012;223:199-210.

33. Cox RW. AFNI: software for analysis and visualization of functional magnetic resonance neuroimages. Comput Biomed Res. 1996;29:162-73.

34. Tziortzi AC, Haber SN, Searle GE, Tsoumpas C, Long CJ, Shotbolt $P$, et al. Connectivity-based functional analysis of dopamine release in the striatum using diffusion-weighted MRI and positron emission tomography. Cereb Cortex. 2014;24:1165-77.

35. Eklund A, Nichols TE, Knutsson H. Cluster failure: why fMRI inferences for spatial extent have inflated false-positive rates. Proc Natl Acad Sci. 2016;113:7900-05.

36. Söderpalm A, Nikolayev L, de Wit H. Effects of stress on responses to methamphetamine in humans. Psychopharmacology. 2003;27:1270-7.

37. Wachtel SR, Ortengren A, de Wit $\mathrm{H}$. The effects of acute haloperidol or risperidone on subjective responses to methamphetamine in healthy volunteers. Drug Alcohol Depend. 2002;68:23-33.

38. Uftring SJ, Wachtel SR, Chu D, McCandless C, Levin DN, de Wit H. An fMRI study of the effect of amphetamine on brain activity. Neuropsychopharmacology. 2001;25:925-35.

39. Eikelboom R, Stewart J. Conditioning of drug-induced physiological responses. Psychol Rev. 1982;89:507-28.

40. Heal DJ, Smith SL, Gosden J, Nutt DJ. Amphetamine, past and present - a pharmacological and clinical perspective. J Psychopharmacol. 2013;27:479-96.

41. Moeller SJ, Honorio J, Tomasi D, Parvaz MA, Woicik PA, Volkow ND, et al. Methylphenidate enhances executive function and optimizes prefrontal function in both health and cocaine addiction. Cereb Cortex. 2014;24:643-53.

42. Nestler, EJ, Hyman, SE. \& Malenka, RC. Chapter 13: Higher cognitive function and behavioral control. 2nd ed. In: Molecular neuropharmacology: a foundation for clinical neuroscience. New York, NY: McGraw-Hill Medical; 2009. P 313-34..

43. Tomasi D, Volkow N, Wang G, Wang R, Telang F, Caparelli E, et al. Methylphenidate enhances brain activation and deactivation responses to visual attention and working memory tasks in healthy controls. Neurolmage. 2011;54:3101-10.

44. Rheker J, Winkler A, Doering BK, Rief W. Learning to experience side effects after antidepressant intake - results from a randomized, controlled, double-blind study. Psychopharmacology. 2017;234:329-38.

45. Koob GF, Volkow ND. Neurobiology of addiction: a neurocircuitry analysis. Lancet Psychiatry. 2016;3:760-73.

46. Ghaziri J, Tucholka A, Girard G, Houde JC, Boucher O, Gilbert G, et al. The corticocortical structural connectivity of the human insula. Cereb Cortex. 2017:27:1216-28.

47. Cavallo JS, Ruiz NA, de Wit H. Extinction of conditioned responses to methamphetamine-associated stimuli in healthy humans. Psychopharmacology. 2016;233:2489-502. 